\title{
NASA ACC High Energy Dynamic Impact Methodology and Outcomes
}

\author{
Kenneth J. Hunziker. ${ }^{1}$ and Jenna K. Pang ${ }^{1}$ \\ Boeing Research \& Technology, Tukwila, WA, 98108 \\ Matthew E. Melis ${ }^{2}$ and J. Michael Pereira ${ }^{3}$ \\ NASA Glenn Research Center, Cleveland, OH, 44135 \\ and \\ Mostafa Rassaian ${ }^{4}$ \\ Boeing Research \& Technology, Tukwila, WA, 98108
}

Subject Category: Structures, Progressive Damage Analysis, Impact

\begin{abstract}
For High Energy Dynamic Impact (HEDI) events, testing to evaluate the structural response of primary aircraft structure for design and certification is both expensive and time consuming. This paper discusses current work seeking to assess, develop, and validate appropriate analytical models that accurately predict physical response, damage, and failure modes for large scale composite structures in HEDI events. Four state-of-the-art Progressive Damage Analysis (PDA) methods were employed for this phased project: LS-DYNA MAT162, LS-DYNA MAT261, Smoothed Particle Galerkin (SPG), and EMU Peridynamics. Multiple material systems were considered, namely T700/5208 textile-infusion triaxial braid, T800/AMD-825 textile-infusion triaxial braid, IM7/8552 uni-directional tape, and SPG 196PW/8552 plain-weave fabric. Extensive ballistic impact testing was performed to support this activity and measured results were compared to predictive models for assessment using panel delamination, panel displacement, force at the load cells, and threshold velocity as measures. Ultimately, the work under this activity provided significant progress in advancing the state-of-the-art in the use of PDA for HEDI events. Each material model had favorable performance comparing to test in some parameters and needed improvement in others. With the lessons learned from this activity, significant progress was made in the ability to predict panel behavior for a more general case beyond the flat panel in a ballistic impact event. Subsequent Phase II of the NASA ACC HEDI effort will continue to build on the coupon testing, flat panel ballistic impact testing, and analysis performed to-date with application of the PDA methods for intended material selections to test articles with greater complexity of configuration, curvature, and scale. It is not the intention of this paper to present a full set of data, but rather to give an overview of the NASA HEDI effort and show a small representative subset of the test and analysis results.
\end{abstract}

\footnotetext{
${ }^{1}$ Structural Analysis Engineer, Structures Technology, 9725 East Marginal Way South, MC 42-56, Tukwila, WA 98108, MC: 42-25, Non-Member.

2 Aerospace Engineer, Structural Mechanics Branch, 21000 Brookpark Road, MS 49-8, Cleveland, OH 44135, AIAA non-member.

${ }^{3}$ Aerospace Engineer, Structural Dynamics Branch, 21000 Brookpark Road, MS 49-8, Cleveland, OH 44135, AIAA Non-member.

${ }^{4}$ Boeing Technical Fellow, Structures Technology, 9725 East Marginal Way South, MC: 42-56, Tukwila, WA 98108, AIAA Associate Fellow.
} 


\section{Introduction}

The NASA Advanced Composites Consortium (ACC) seeks to develop and transition technology that will enable a reduction in the timeline required for development and certification of new aircraft structure that utilize advanced composite materials. The consortium is organized around three technical challenges: Predictive capabilities, Rapid Inspection, and Manufacturing Process Simulation. The first, Predictive Capabilities, intends to develop robust analysis methods that can enable smarter physical testing and improve preliminary tool designs. Smarter testing and better preliminary tools allow for a reduction in costly large-scale testing and potentially the number of redesigns. For High Energy Dynamic Impact (HEDI) events, a lack of fully validated and standardized analysis approaches has led to the increased use of testing to evaluate the structural response of primary aircraft structure for design and certification. The component testing required for HEDI is both expensive and time consuming due to the size, complexity, and wide range of possible design configurations. The current work seeks to assess, develop, and validate appropriate analytical models that accurately predict physical response, damage, and failure modes for large scale composite structures in HEDI events. This paper is organized into five main sections. After the introduction, an overview of the phased approach to the HEDI technical development is presented followed by details of the ballistic panel testing, test-analysis comparison, and finally some concluding statements. It is not the intention of this paper to present a full set of data, but rather to give an overview of the NASA HEDI effort and show a small representative subset of the test and analysis results. The coupon and panel testing and analysis efforts are described in detail in other papers at this technical interchange.

\section{High Energy Dynamic Impact (HEDI) Development Overview}

High fidelity analysis methods known as Progressive Damage Analysis (PDA) methods, capable of reliably predicting the onset and progression of damage in composite materials are being developed for HEDI event simulation. Four state-of-the-art PDA methods are being investigated: LS-DYNA MAT162, LS-DYNA MAT261, Smoothed Particle Galerkin (SPG), and EMU Peridynamics. Validation of these PDA models aims to follow a building block approach, starting with coupon- and component-level development. As part of the overall effort, material characterization testing was performed to bridge gaps between existing experimental data and the material property inputs required to predict ballistic impact behavior at the component-level. The material models were updated with the results of the coupon-level testing and were used to generate pre-test predictions of panel behavior and damage in ballistic impact events. To assess the accuracy of the PDA methods, these pre-test predictions were compared to the results of ballistic impact testing. Figure II-1 illustrates the steps taken in the technical development of the selected PDA methods including preliminary evaluation of the state-of-the-art methods. Detailed descriptions of LS-DYNA MAT162, LS-DYNA MAT261, and EMU Peridynamics modeling approaches including some impact test-analysis correlations are presented in Ref. 1, 2, 3. In support of the PDA method validation activity, eighty-four (84) flat component-level panels were ballistically impacted at NASA Glenn Research Center (GRC). The test activities were designed, performed, and analyzed in collaboration by ACC participants (NASA, The Boeing Company, United Technologies/Pratt \& Whitney, and General Electric Aviation) and captured the effects of a

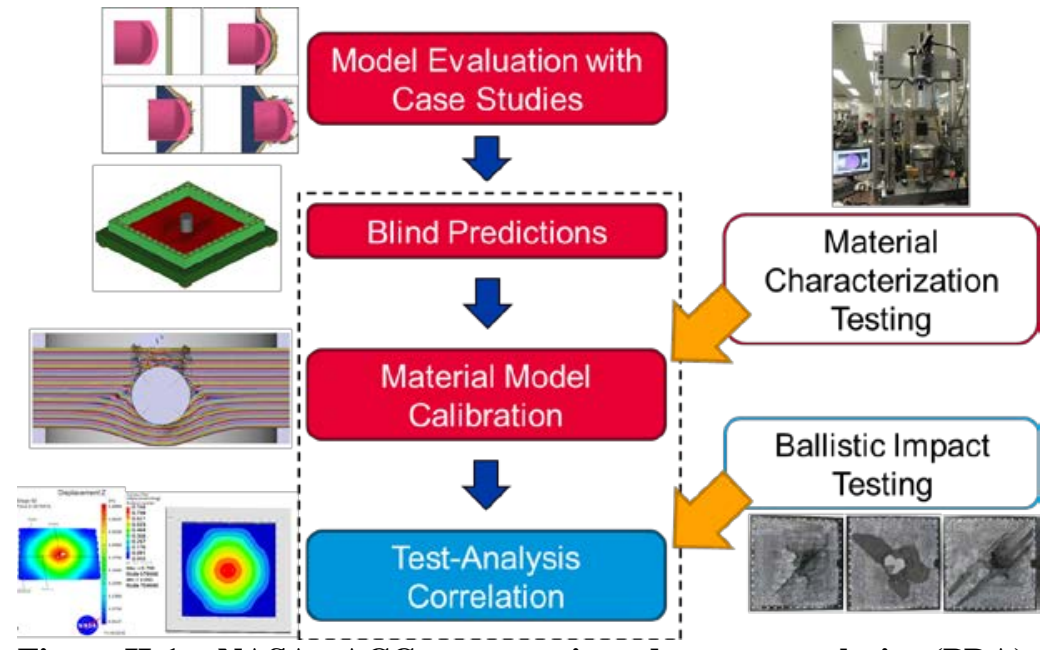

Figure II-1. NASA ACC progressive damage analysis (PDA) method technical develonment. wide variety of impact variables including impact velocity, projectile type, laminate thickness, fiber architecture and material.

Four material types were selected as representative of materials potentially used for structures impacted in HEDI events, such as engine casing and fuselage shielding; selected materials were T700/5208 textile-infusion triaxial 
braid, T800/AMD-825 textile-infusion triaxial braid, IM7/8552 uni-directional tape, and SPG 196-PW/8552 plain-
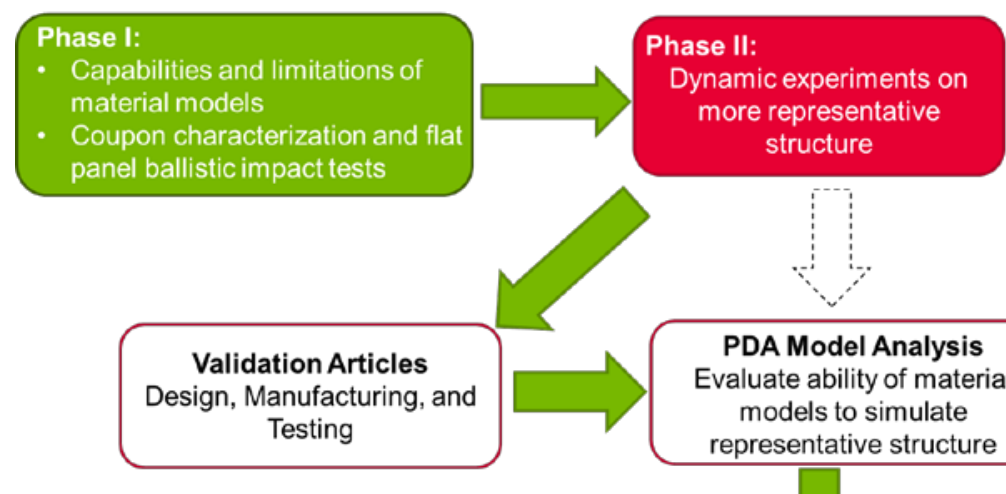

PDA Model Analysis

Evaluate ability of material models to simulate representative structure

GOAL: Develop analytical models that accurately predict physical

response, damage, and failure modes of large composite structures. Limitations and "best practices" documented

Figure II-2. NASA ACC HEDI technical approach. weave fabric.

These ballistic impact tests were performed and material types were evaluated as part of Phase I of the NASA ACC HEDI project. The main objective of Phase I was to assess the capabilities and limitations of the state-of-the-art PDA methods with realistic impact events, as represented by simple flat panel ballistic impact tests. Phase II of the program continues PDA assessment and development by adding representative features to the flat panels and testing in a similar manner to Phase I representative of fuselage structure shielding and engine containment. The ultimate goal for this phased project is the development of analytical models

with accurate predictive methods for high-energy impact events against a variety of structures, configurations, and design parameters. Figure II-2 shows the overall technical approach for the NASA ACC HEDI project leveraging Phases I and II to a full technology demonstrator.

Under Phase I of the NASA ACC HEDI project, ballistic impact testing was performed on flat component-level test articles. In total, eighty-four (84) ballistic impact tests were performed at NASA Glenn Research Center (GRC) in the Ballistic Impact Lab. Phase II of the program is intended to build on the non-configured flat panels tested in Phase I with the inclusion of configuration (fastener, stringer, frame), curvature, and scale. Figure III-1 outlines the overall test plan for the NASA ACC HEDI program as it increases complexity as it moves up the length-scale.

\section{A. Test Fixture}

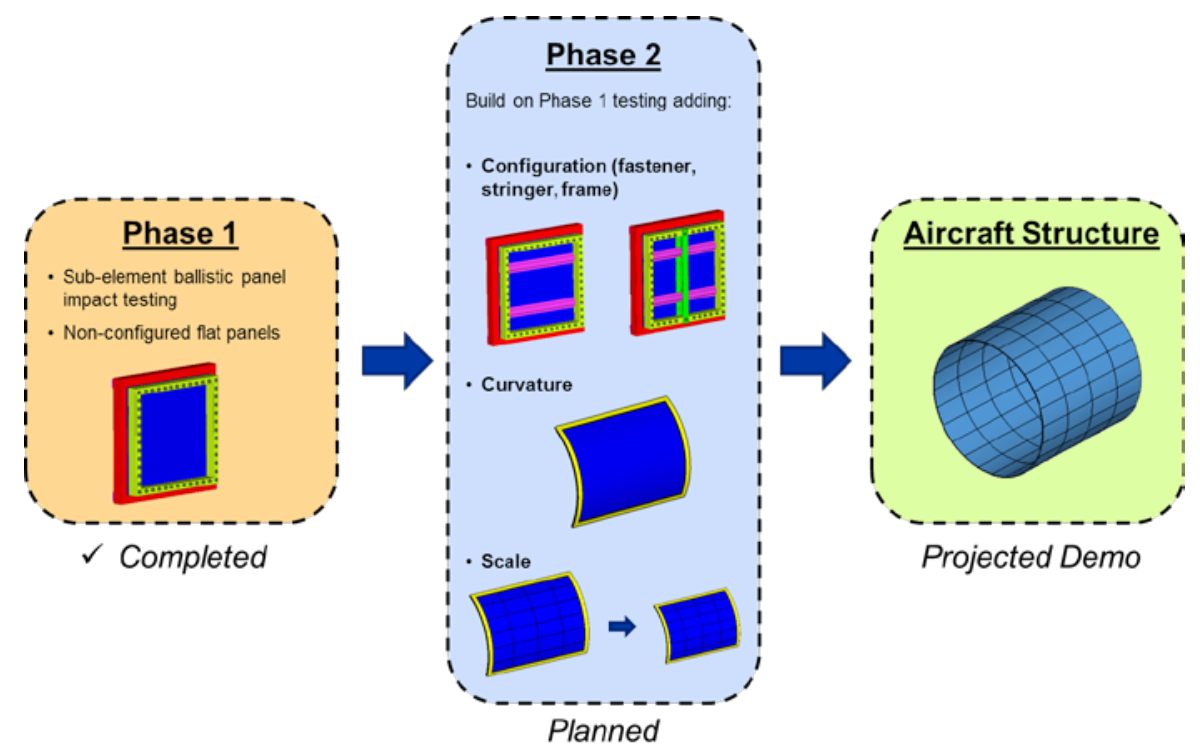

Figure III-3. Overview of NASA ACC HEDI testing.

The Phase I test article consisted of a 25" x 25" panel secured to two square picture-frames and connected to the upright supports through four piezoelectric load cells (shown in Fig. III-2). The load cells isolated the frame from the supports and were sampled at a rate that captured the dynamic impulse. Each load cell had a full scale of 80,000 lb and was preloaded to a nominal value of $16,000 \mathrm{lb}$. 


\section{B. Test Setup}

Phase I impact testing consisted of three unique projectiles called "blunt", "sharp", and the Pratt \& Whitney projectile. The blunt projectile consisted of a cylinder with 3" diameter and 3.5" length made of the rubber compound Flexane ${ }^{\circledR} 94$ - shown in Fig. III-3 (a). The sharp projectile was an assembly consisting of a 2" x 2" x 0.25" titanium insert embedded in Flexane ${ }^{\circledR} 94$ and encased in a hard foam sabot - shown in Fig. III-3 (b). The Pratt \& Whitney projectile was a Ti-6Al-4V cylinder with a hemispherical nose - shown in Fig. III-3 (c). The projectile was propelled into the test specimen using a single stage gas gun with nitrogen gas as the propellant and was released with the use of a Mylar® burst valve. The gas gun had a 3" inner diameter and 23' length with a 1900 in $^{3}$ pressure vessel volume. Digital image correlation (DIC) was used to capture and validate test data such as projectile impact velocity, projectile residual velocity, and panel deflection.

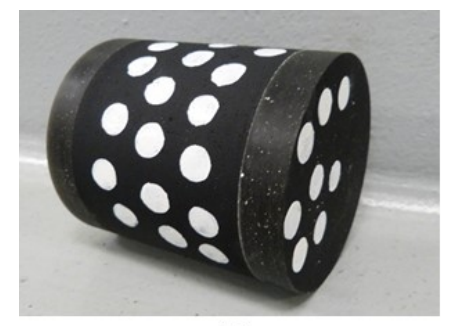

(a)

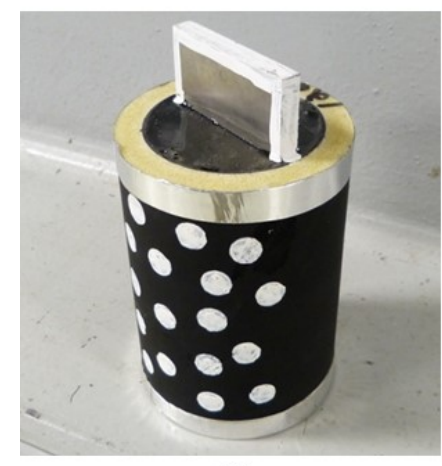

(b)

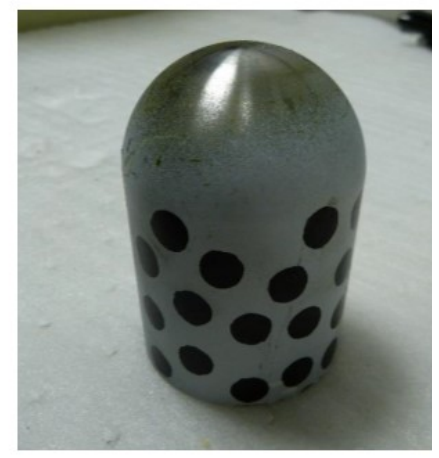

(c)

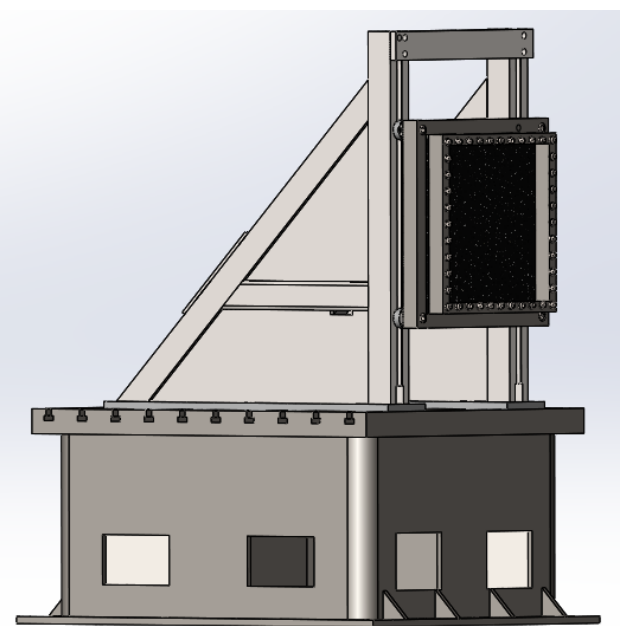

Figure III-4. Model of ballistic impact test fixture developed by NASA Glenn Research Center.

The test setup at the NASA Glenn Ballistic Impact Lab is shown in Figure III-4. Photographic images were taken using a set of stereo high speed cameras capable of fully 3D Digital Image Correlation (DIC) for both quantitative and qualitative data.

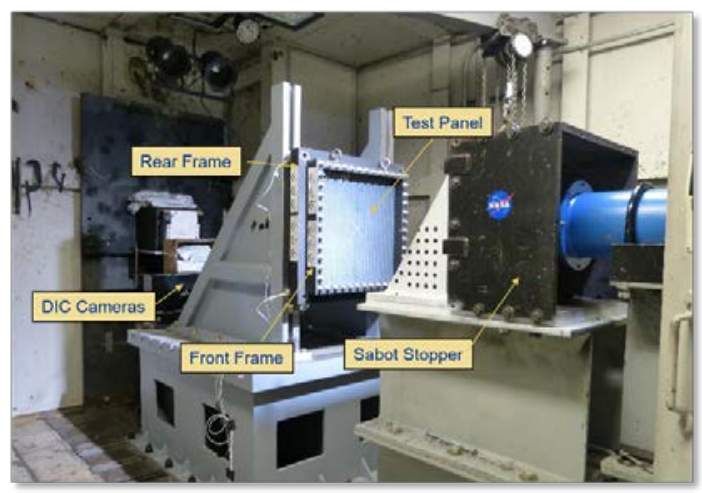

Figure III-6. Impact test setup at NASA Glenn Ballistic Impact Lab.

Figure III-5. (a) "Blunt” projectile (b) "Sharp” projectile (c) Pratt and Whitney projectile - all with digital image correlation markers.

\section{Test Results}

Using DIC, a set of data was collected for each impact test. The projectile velocity and orientation while in flight were measured using a pair of high speed cameras. Displacements and strains on the rear surface of the panel were computed using the commercial software ARAMIS in conjunction with a calibrated pair of high speed cameras focused on the back side of the panel. Pictures were taken of the front and the rear of the panel to catalogue visible panel damage; additional data was collected using ultrasonic techniques to identify damage in the material such as delamination with non-destructive inspection (NDI). 
A representative subset of the testing results is shown through post-test images in Fig. III-5 and Fig. III-6. The test articles are a series of IM7/8552 unidirectional tape, fabric, and hybrid tape-fabric laminates. Figure III-5 shows rear-view images of the impacted panel damage for the blunt projectile with initial impact velocities of $660-670 \mathrm{ft} / \mathrm{s}$ and Fig. III-6 details the NDI results for the same set of panels.

Overall, the severity of visible damage increased as impact velocity increased. Post-test NDI indicated panels with rebounding projectiles had large panel-wide delaminations that first occurred at the same relative thickness, a different failure mode than panels with penetrating projectiles which showed fiber breakage as well as localized and regional delaminations at varying panel depths. Test case LG1220 in Figure III-6, a rebounding event, is an example of this divergent behavior when compared to other images in the set.
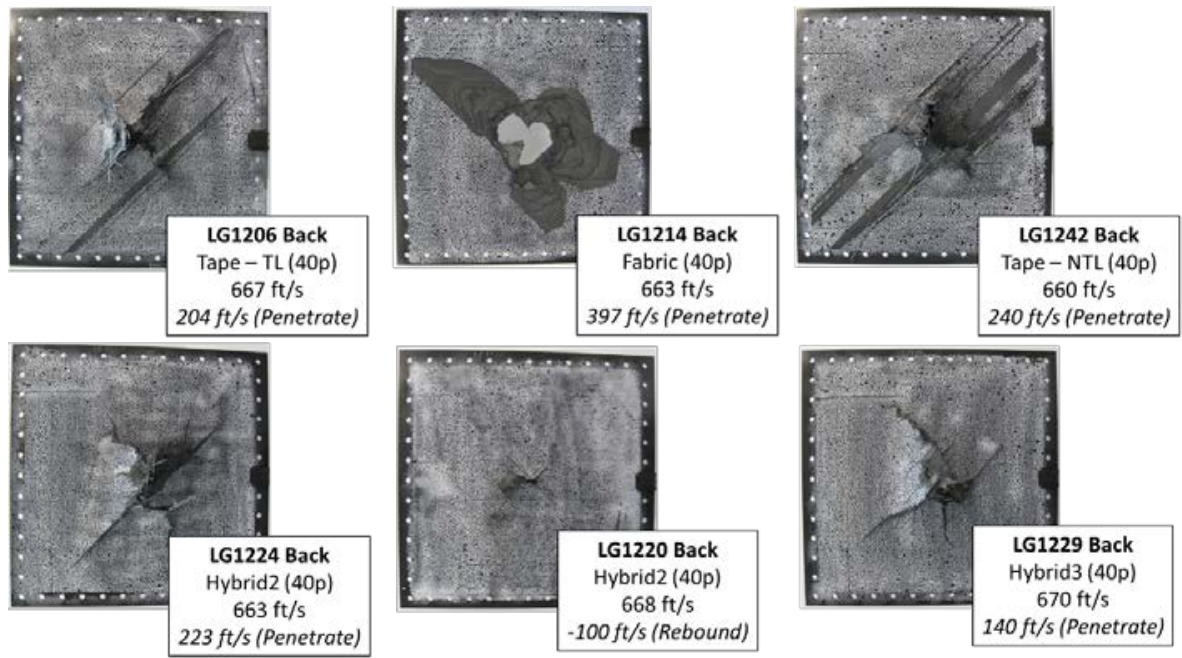

Figure III-7. Backside post-test images of 40-ply panels impacted with a blunt projectile.

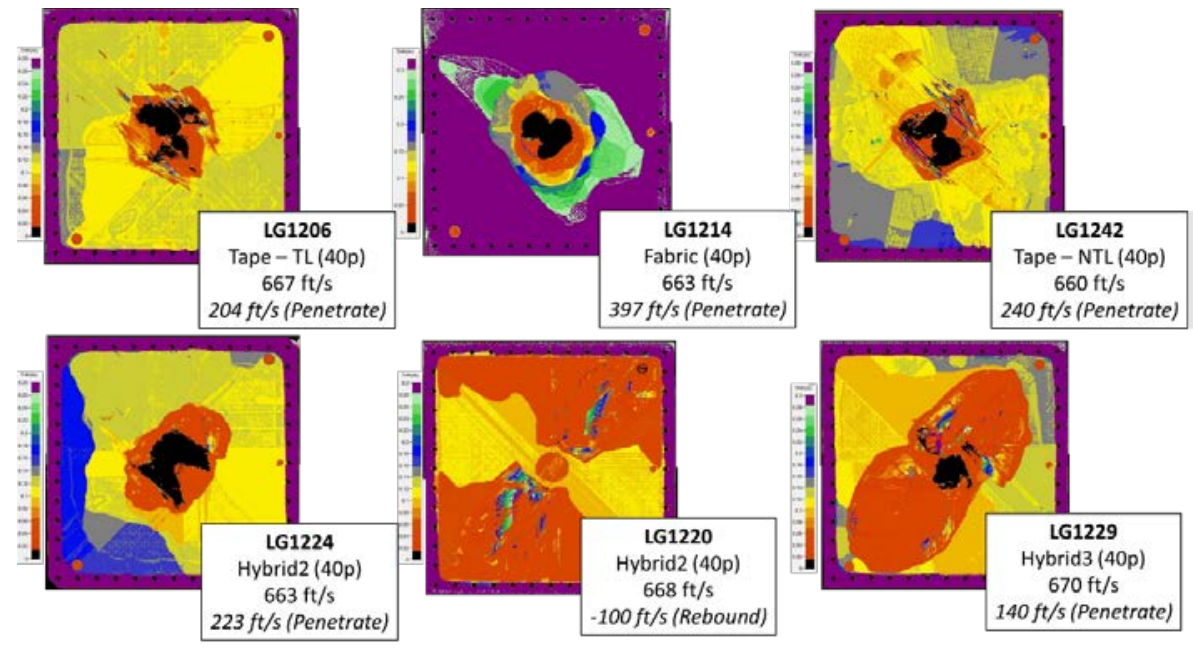

Figure III-8. Time of flight NDE data for 40-ply panels impacted with a blunt projectile.

NDI also indicated that

the fabric panels featured material tear-off damage which is a distinctly different damage pattern than tape and hybrid panels which showed significant through-thickness fiber damage. Fabric panels also exhibited damage that was narrower around the point of impact than other panels. Test case LG1214 in Fig. III-6 is a striking example of this observation.

\section{Test-Analysis Comparison}

Four material models were used for this analysis activity: LS-DYNA MAT162/MAT261, Smoothed Particle Galerkin (SPG), and EMU Peridynamics. Each material model was chosen to predict the behavior of composite panels under the highly complex and progressive damage scenario seen in a high energy impact event. LS-DYNA MAT162 relies on a continuum mecahnics approach to progressive damage whereas LS-DYNA MAT261 uses a fracture-mechanics approach. SPG is a discrete particles formulation with progressive damage and failure and EMU Peridynamics is a mesh-free (i.e., discrete particles), non local method. The remainder of this section discusses the comparison between ballistic impact test and simulation using four measures: panel delamination, panel displacement, force at the load cells, and threshold velocity $\left(\mathrm{V}_{50}\right)$. Figure IV-1 shows the four test-analysis comparison measures for a selected test case. 


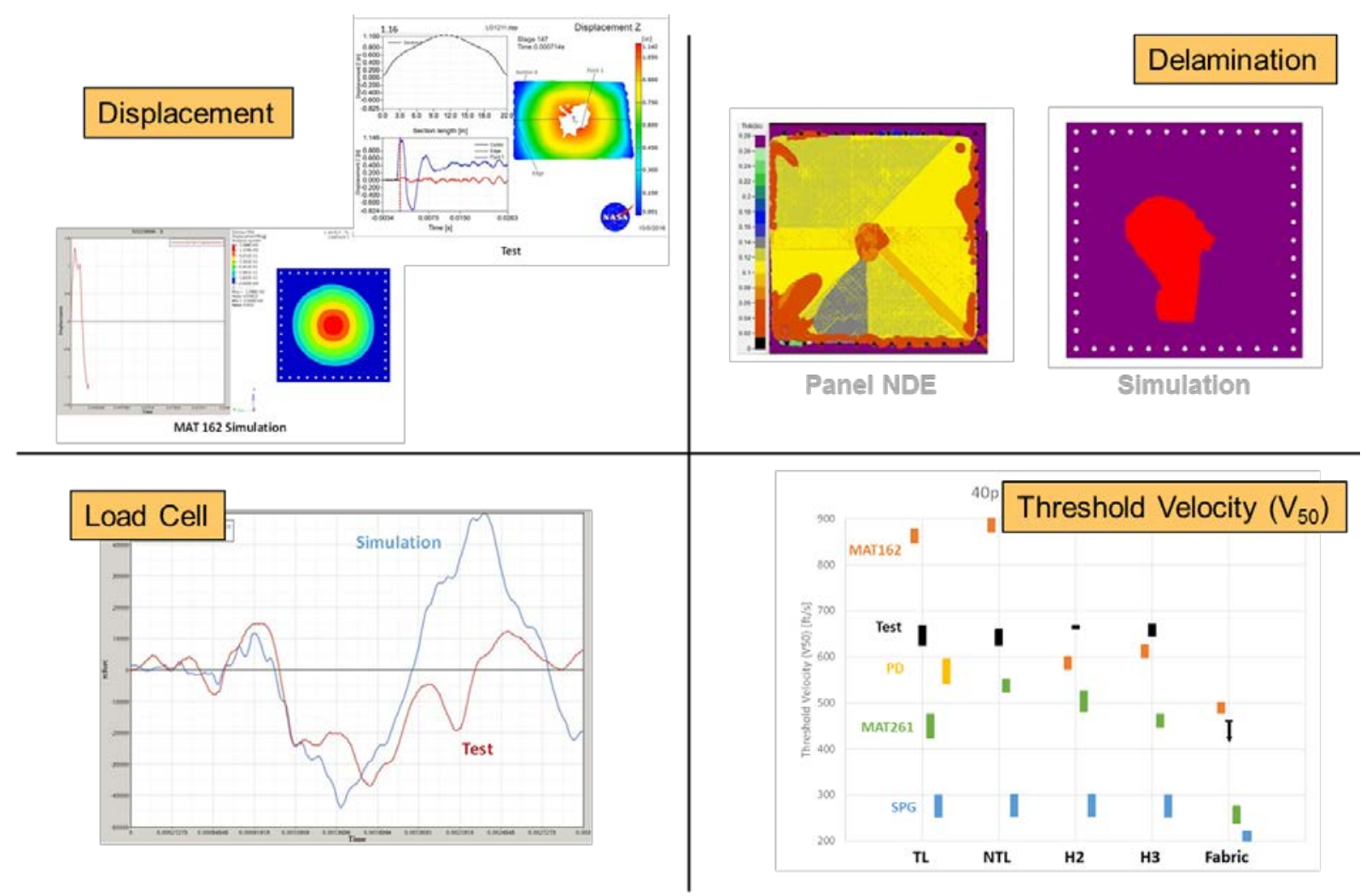

Figure IV-1. Test-analysis correlation for selected test cases.

The upper right corner of Fig. IV-1 compares the NDI results observed in the test panel and those predicted by simulation after impact for LS-DYNA MAT162. For this specific case, the NDI results show considerable fiber damage that is not predicted by the simulation. Note that the contour colors in the NDE image represent delamination depth information whereas the simulation image only provides an outline of the damage region.

The upper left hand corner of Fig. IV-1 shows a displacement comparison; from analysis, the lateral displacement time-history of the panel center is shown next to a contour image at the maximum value and is compared with similar test displacements in the lower half of the image. There are a number of points labeled on the test panel image that correlate to the time-history chart; these points were selected on a per-test basis for quality of data but generally include center, near center, and edge points. The image above the time-history indicates the displacement of the horizontal centerline at the current time step and was used to extrapolate the displacement of the center point in the event that the DIC data was incomplete. It should be noted that the simulation and test displacement time-histories might not be taken at the exact same location. Often, damage to the back of the panel prevented data collection at the point of maximum displacement so an undamaged location was chosen. However, there is sufficient data to compare the nature of the time-histories and the magnitude using the contour plot. Both the simulation displacement magnitude (from the contour plot) and time-history compare favorably with test data.

Load cell data was collected during the test and compared with corresponding data taken from the simulations as shown in the bottom left of Fig. IV-1. The raw data was modified slightly based on a fixed time point to provide a more consistent comparison. It is important to acknowledge that the test-analysis comparison should only consider up to the max compressive section of the load time history plots as the tension value has no physical meaning due to the pre-load in the panel. Simulation and test load cell data were observed to match quite well approximately for the first $2 \mathrm{~ms}$ of the impact event, particularly the magnitude of the peak compressive load. This time period corresponds to all of the observed damage in the simulation and later effects are considered secondary.

The bottom right corner of Fig. IV-1 compares threshold velocity, V50, for LS-DYNA MAT162, MAT261, SPG, and EMU Peridynamics simulations and test. For this specific case LS-DYNA MAT162 looks to over-predict the $\mathrm{V}_{50}$ whereas the other material models under-predicted the test data with similar trends. Across the different material models, this measure gives the best quantitative comparison with test but does not consider the type and extent of damage. 
The following figures show still images of representative rebound and penetration impact events for the blunt projectile type. Figure IV-2 shows a typical blunt projectile response to a rebound and penetration event. These images were chosen to demonstrate behavior that is representative of a broad selection of tests and different projectile velocities, panel thicknesses, and material models may yield different results.

As shown in Fig. IV-2, the behavior of the Flexane material used in the Phase I blunt projectile was extremely complex, particularly in a dynamic impact event. The projectile has been shown to be hyperelastic, highly strain-rate dependent, and viscoelastic, of which the latter two were not accounted for in the material model. The complexity of the material caused the projectile response to affect the duration of impact, peak force exerted on panel, rate of momentum transfer, and contact area with panel. Ultimately, the projectile model showed behavior in simulation that was not observed in test and warrants further investigation. The behavior of the projectile likely impacts the comparison between test and analysis and bridge testing and analysis is planned before further testing is conducted. Additionally, the current work under the NASA ACC HEDI Phase II effort is planned to include a projectile with less complex

behavior.

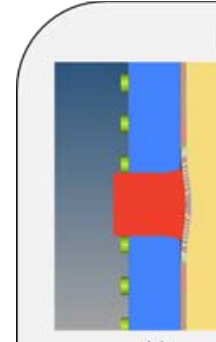

(1)

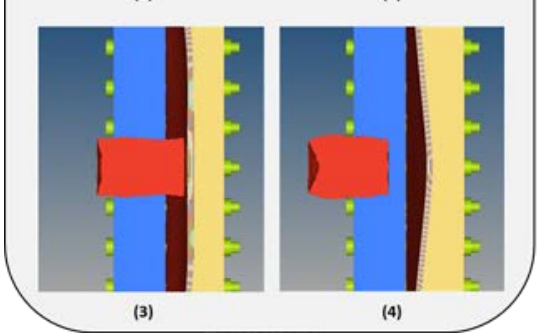

Figure IV-2. Typical response of a blunt projectile for a rebound event and a penetration event.

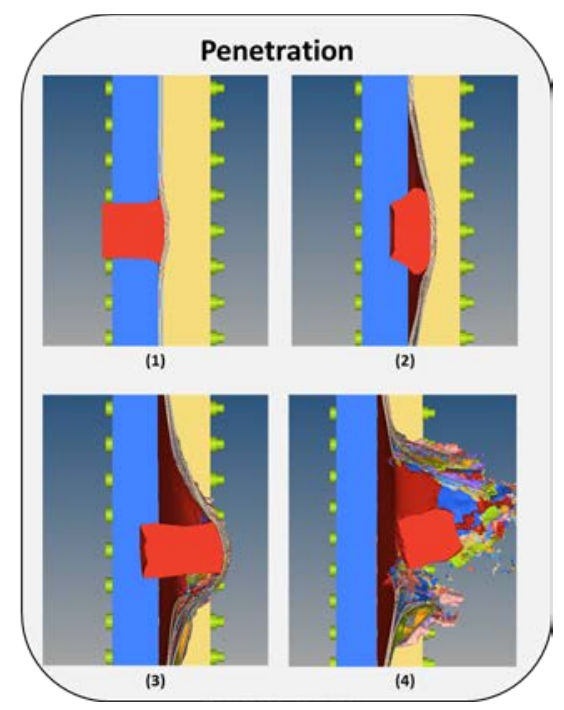

\section{Conclusion}

The HEDI Consortium Research Team (CRT) made significant progress in advancing the state-of-the-art in the use of progress damage analysis (PDA) for high energy dynamic impact (HEDI) events. Overall, each material model compared favorably with test in some categories and had room for improvement in others. The LS-DYNA MAT162 and 261 material models showed good agreement between predictions and test results in both deflection time history and damage with significant predictive deviations from measured $V_{50}$ threshold velocity in most cases. Peridynamics showed similarly promising results as LS-DYNA MAT261 and MAT162, with particular success in accurately predicting $V_{50}$. The initial evaluation for SPG indicates it is not currently suitable for modeling high energy dynamic impact due to premature out-of-plane shear failure leading to low $\mathrm{V}_{50}$; development continues for other applications.

Members of the CRT conducted extensive coupon characterization testing and ballistic impact testing with subelements to furnish data for calibration of the selected state-of-the-art progressive damage analysis (SoA-PDA) methods/material models and assessment of Progressive Damage Analysis (PDA) model prediction performance. A major finding from the completed effort is the need for further work to characterize the behavior of the projectiles used in Phase I in order to close the gap between the comparison between test and analysis.

Ultimately, the work under this activity provided technical development of PDA models for a wide range of impact applications such as bird or hail impact, thrown threat, blade out, etc. With the lessons learned from this activity, significant progress was made in being able to predict panel behavior for a more general case beyond the flat panel in a ballistic impact event. Subsequent Phase II of the NASA ACC HEDI effort will continue to build on the coupon testing, flat panel ballistic impact testing, and analysis performed in Phase 1 with application of the PDA methods to test articles with greater complexity of configuration, curvature, and length scale that represent realistic aerospace structures. 


\section{Acknowledgements}

The material in this paper is based upon work supported by NASA under Award No. NNL09AA00A. Any opinions, findings, and conclusions or recommendations expressed in this material are those of the author(s) and do not necessarily reflect the views of the National Aeronautics and Space Administration.

\section{References}

${ }^{1}$ Molitor, M., Justusson, B., Pang, J., and Rassaian, M. , “Comparison of Test Methods to Determine Failure Parameters for MAT162 Calibration,” AIAA SciTech Forum, Kissimmee, FL, 8-12 Jan. 2018 (submitted for publication).

${ }^{2}$ Byar, A., Iqbal, J., Pang, J., and Rassaian, M., “Determination of Ballistic Limit for IM7/8552 Using MAT261,” AIAA SciTech Forum, Kissimmee, FL, 8-12 Jan. 2018 (submitted for publication).

${ }^{3}$ Weckner, O., Cuenca, F., Silling, S. A., Rassaian, M., and Pang, J., "Determination of Ballistic Limit for IM7/8552 Using Peridynamics,” AIAA SciTech Forum, Kissimmee, FL, 8-12 Jan. 2018 (submitted for publication). 